\title{
A Comprehensive Overview on the Formation of Homomorphic Copies in Coset Graphs for the Modular Group
}

\author{
Hanan Alolaiyan $\mathbb{D D}^{1}$ Abdul Razaq $\mathbb{D}^{2},{ }^{2}$ Awais Yousaf $\mathbb{D}^{3},{ }^{3}$ and Rida Zahra ${ }^{4}$ \\ ${ }^{1}$ Department of Mathematics, King Saud University, Riyadh, Saudi Arabia \\ ${ }^{2}$ Department of Mathematics, Division of Science and Technology, University of Education Lahore, Lahore, Pakistan \\ ${ }^{3}$ Department of Mathematics, The Islamia University of Bahawalpur, Bahawalpur 63100, Pakistan \\ ${ }^{4}$ Department of Mathematics, Govt. Girls Elementary School Narri, Khushab, Pakistan \\ Correspondence should be addressed to Abdul Razaq; abdul.razaq@ue.edu.pk
}

Received 9 August 2021; Accepted 3 September 2021; Published 21 September 2021

Academic Editor: Kenan Yildirim

Copyright (C) 2021 Hanan Alolaiyan et al. This is an open access article distributed under the Creative Commons Attribution License, which permits unrestricted use, distribution, and reproduction in any medium, provided the original work is properly cited.

\begin{abstract}
This work deals with the well-known group-theoretic graphs called coset graphs for the modular group $G$ and its applications. The group action of $G$ on real quadratic fields forms infinite coset graphs. These graphs are made up of closed paths. When $M$ acts on the finite field $Z_{p}$, the coset graph appears through the contraction of the vertices of these infinite graphs. Thus, finite coset graphs are composed of homomorphic copies of closed paths in infinite coset graphs. In this work, we have presented a comprehensive overview of the formation of homomorphic copies.
\end{abstract}

\section{Introduction}

The study of groups via their actions has been a central theme in group theory, since the completion of the classification of simple groups in the 1980s. This most commonly takes the form of actions on vector spaces and similar commutative objects or on more elementary combinatorial objects. It is not an exaggeration to say that the modular group $G$ (see [1-4]) is the single most important infinite discrete group, through its myriad connections with number theory, geometry, and topology. There is a long and venerable history of studying its actions, particularly on finite sets, which goes back to before the turn of the twentieth century. The modular group $G$ has two generators $f$ and $g$, where $f: x \longrightarrow-1 / x$ and $g: x \longrightarrow x-1 / x$ are linear fractional transformations. The finite presentation of $G$ is $\langle f, g$ : $f^{2}=g^{3}=1>$. It means that it is a free product of $C_{2}$ and $C_{3}$. The linear fractional transformation $h: x \longrightarrow 1 / x$ extends $G$ to $G^{\prime}$ because it inverts $f$ and $g$; that is, $h^{2}=(f h)^{2}=(g h)^{2}=1$. Thus, extended modular group $G^{\prime}$ has three generators $f, g$, and $h$ and its finite presentation is $\left\langle f, g, h: f^{2}=g^{3}=h^{2}=\right.$ $(f h)^{2}=(g h)^{2}=1>$.
Graph theory has applications in various branches of mathematics [5, 6]. Several topological and algebraic structures can be studied in a more effective way by using graphs. Graphical techniques are specifically utilized to investigate the finitely generated groups. The graphs prove to be an effective and simple method to solve many mathematical problems [7-9].

The use of graphs to represent group actions has a venerable history. Cayley [8] published the first work on this topic. Mathematicians like Coxeter [9], Burnside [10], Stothers [11], Everitt [12], Conder [13], Whitehead [14], and others provided pioneering works on graphical representations of groups. The action of a modular group on certain objects can be represented by a certain type of graphs, called coset graphs. These were introduced by Higman in 1978. Later, in 1983, Mushtaq [15] laid their foundation. These graphs consist of triangles connected to each other. The edges of triangles are permuted anticlockwise to represent by g. Each vertex of a triangle is connected by $f$ to another vertex of the triangle (which may be the same triangle).

Moreover, the vertices of the coset graph that are fixed by $f$ and $g$ are represented by heavy dots. Since $(g h)^{2}=1$ implies 


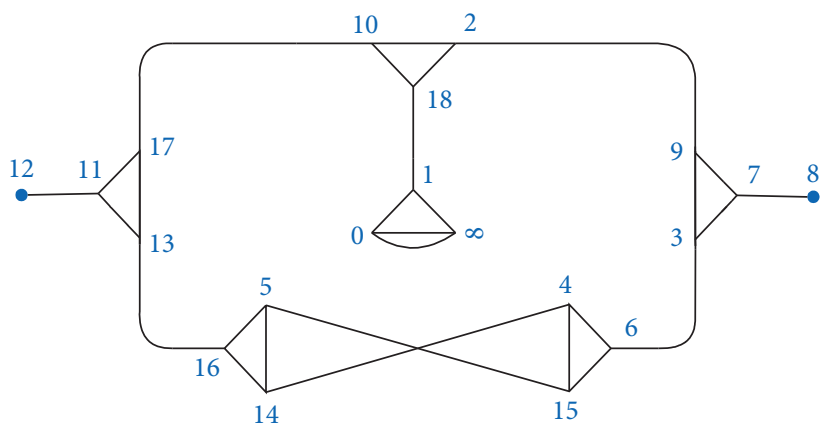

Figure 1: The coset graph of $Z_{19} \cup\{\infty\}$.

$h g h^{-1}=\mathrm{g}^{-1-1}, h$ turns around the direction of the triangles like reflection. Thus, we do not introduce $h$-edges in coset graphs, so that they remain simple.

The action of $G^{\prime}$ on finite field $Z_{p}$ is not possible because $f$ maps 0 to $\infty$. Thus, we add $\infty$ to $Z_{p}$ in order to make the action possible.
Example 1. Let us consider the action of modular group on $Z_{19} \cup\{\infty\}$. The permutation representations $f, g$, and $h$ are

$$
\begin{aligned}
& f:(0, \infty)(13,16)(1,18)(11,12)(2,9)(10,17)(3,6)(7,8)(4,14)(15,5), \\
& g:(\infty, 1,0)(10,18,2)(7,9,3)(15,6,4)(16,14,5)(17,11,13)(8)(12), \\
& h:(0, \infty)(10,2)(13,3)(5,4)(16,6)(11,7)(12,8)(17,9)(15,14)(1)(18) .
\end{aligned}
$$

The corresponding coset graph is shown in Figure 1.

Definition 1. Let $\Gamma$ and $\Gamma^{\prime}$ be two coset graphs; then $\Gamma^{\prime}$ is a homomorphic copy of $\Gamma$ if

(i) order of $\Gamma^{\prime}$ is less than the order of $\Gamma$, that is, $\left|\Gamma^{\prime}\right|<|\Gamma|$,

(ii) $u$ is a vertex in $\Gamma$ such that $(u) x=u$ for some $x \in G$, and then there exists some vertex $u^{\prime} \in \Gamma^{\prime}$ such that $\left(u^{\prime}\right) x=u$.

Let $q^{\prime}$ be a real quadratic irrational number; then $q^{\prime}=((\alpha+\sqrt{m}) / \beta)$, where $m$ is a square-free natural number and $\left(\alpha,\left(\left(\alpha^{2}+m\right) / \beta\right), \beta\right)=1$. In [16], Mushtaq studied the group action of $G$ on real quadratic fields and showed that the corresponding coset graphs are infinite. Figure 2 shows a small patch of these graphs.

Due to the emergence of infinite graphs, the action of $G$ on $q^{\prime}=((\alpha+\sqrt{m}) / \beta)$ through coset graphs is not easy to study. Therefore, the action of $G$ on $Z_{p} \cup\{\infty\}$ becomes important. The coset graphs for $Z_{p} \cup\{\infty\}$ are homomorphic copies of the infinite graphs for $q^{\prime}=((\alpha+\sqrt{m}) / \beta)$, where $m \equiv n^{2} \bmod p$, for any natural number $n$. For example, the coset graph shown in Figure 1 is the homomorphic copy of the coset graph for $((\alpha+\sqrt{17}) / \beta)$ because $17 \equiv 6^{2} \bmod 19$.

For further details about coset graphs, we refer the readers to [17-22].

The main contributions of this paper are as follows:

(1) A thorough study on the formation of homomorphic copies of coset graphs is presented
(2) We have developed a formula to compute all homomorphic copies of the closed path of rank 4

\section{Closed Paths in Coset Graph}

Definition 2. A closed path in a coset graph containing a vertex fixed by $(f g)^{m_{1}}\left(f g^{-1}\right)^{m_{2}}, \ldots,(f g)^{m_{k-1}}$ $\left(f g^{-1}\right)^{m_{k}} \in \operatorname{PSL}(2, Z)$, where $m_{1}, m_{2}, \ldots, m_{k} \in N$ is called a closed path of rank $k$. It is denoted by $\left(m_{1}, m_{2}, \ldots, m_{k}\right)$. In [23], it has been proved that the rank of closed paths is always even.

Remark 1. Let $x, y \in G$ and $(u) x=u$. Then vertex $(u) y$ is fixed by $y^{-1} x y$.

Suppose that $u_{1}$ and $u_{2}$ are any two vertices in a closed path $C$, such that $\left(u_{1}\right) x_{1}=u_{1}$ and $\left(u_{2}\right) x_{2}=u_{2}$. Let $\left(u_{1}\right) x_{3}=u_{2}$; then $x_{1}^{-1} x_{3}$ also maps $u_{1}$ to $u_{2}$. Clearly, $x_{3}$ and $x_{1}^{-1} x_{3}$ are the only possible paths to travel from $u_{1}$ to $u_{2}$. By contraction of vertices $u_{1}$ and $u_{2}$, we mean $u_{1}$ and $u_{2}$ merge to form a node $u=u_{1}=u_{2}$ such that $u=u_{1}=u_{2}$ is fixed by both $x_{3}$ and $x_{1}^{-1} x_{3}$. This can be done by making a closed path $C^{\prime}$ containing $u$ such that $(u) x_{3}=u$ and then by applying $x_{1}^{-1} x_{3}$ on $u$ such that $x_{1}^{-1} x_{3}$ ends at $u$. Consequently, a graph $\gamma$ is evolved, which is a homomorphic copy of $C$. Note that, in addition to $u_{1}$ and $u_{2}$, there are some other pairs of vertices in $C$, which also compose $\gamma$ by contraction. In fact, during the formation of $\gamma$ by contracting $u_{1}$ and $u_{2}$, some more pairs also get contracted. How many are they? The following theorems help to calculate this number. 


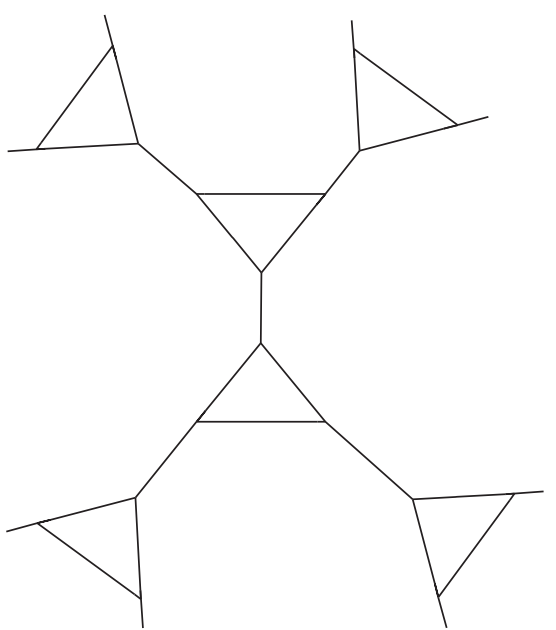

Figure 2: An overview of the small portion of infinite graphs.

Theorem 1 (see [24]). Let a homomorphic copy $\gamma$ of $C$ be formed by contracting its vertices $u_{1}$ and $u_{2}$. Then $\gamma$ is obtainable also by contracting the pair $\left(u_{1}\right) x,\left(u_{2}\right) x$ for some $x \in G$.

Theorem 2 (see [24]). The number of pairs to obtain $\gamma$ is equal to the number of elements $x \in G$, such that $\left(u_{1}\right) x$ and $\left(u_{2}\right) x$ lie in $C$.

Example 2. Consider a closed path (see Figure 3) containing a vertex $v$ which is a fixed point of $(f g)^{4}\left(f g^{-1}\right)^{3}$. Thus, it is a rank two closed path, denoted by $(4,3)$.

Figure 4 represents the homomorphic copy of $(4,3)$ generated through contraction of vertices $u$ and $v$.

\section{Formation of Homomorphic Copies through Contraction of Vertices}

The coset graphs are made up of closed paths. The vertices of infinite graphs are contracted in a specific manner to evolve finite coset graphs. Therefore, a question arises: how many distinct homomorphic copies can be created by contracting all pairs in a closed path? In this work, we have developed a technique to find all homomorphic copies of the closed paths $\left(m_{1}, m_{2}, m_{3}, m_{4}\right)$, where $m_{1}>m_{2}>m_{3}$ and $m_{2}=m_{4}$, in coset graphs. Diagrammatically $\left(m_{1}, m_{2}, m_{3}, m_{4}\right)$, where $m_{1}>m_{2}>m_{3}$ and $m_{2}=m_{4}$, is shown in Figure 5 .

In the remaining part of the paper, we denote the closed path $\left(m_{1}, m_{2}, m_{3}, m_{4}\right)$, where $m_{1}>m_{2}>m_{3}$ and $m_{2}=m_{4}$, by $\psi$. Throughout this paper, the mirror image of any homomorphic copy $\gamma$ is denoted by $\gamma^{*}$. If $x=f g^{\kappa_{1}} f g^{\kappa_{2}}, \ldots, f g^{\kappa_{n}} \in \operatorname{PSL}(2, Z)$, where $\kappa_{i}=1$ or -1 , then let $x^{*}=f g^{-\kappa_{1}} f g^{-\kappa_{2}}, \ldots, f g^{-\kappa_{n}}$. If $x$ fixes any vertex $u$, then the vertex fixed by $x^{*}$ is $u^{*}$.

Remark 2. Since $(g h)^{2}=1$ implies $h g h^{-1}=\mathrm{g}^{-1}, h$ turns around the direction of the triangles like reflection. If $\gamma$ is obtained by contracting vertices $u_{1}$ and $u_{2}$ of any closed path $C$, then the mirror image $\gamma^{*}$ of $\gamma$ can be created by

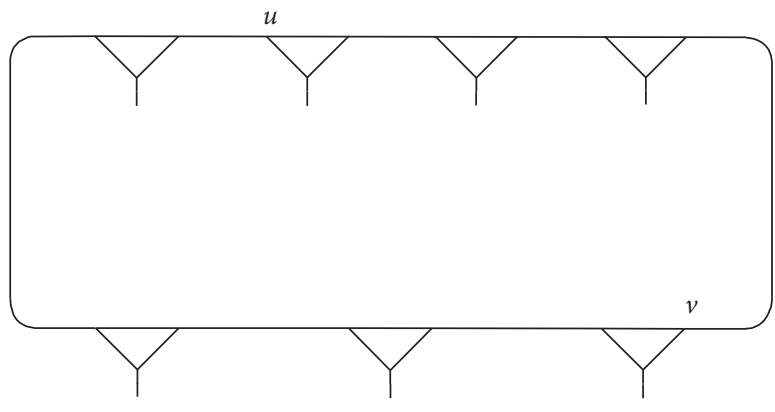

Figure 3: The closed path $(4,3)$.

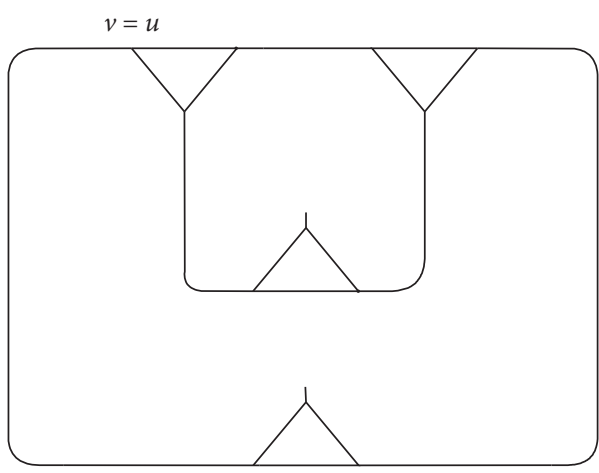

Figure 4: A homomorphic copy of $(4,3)$.

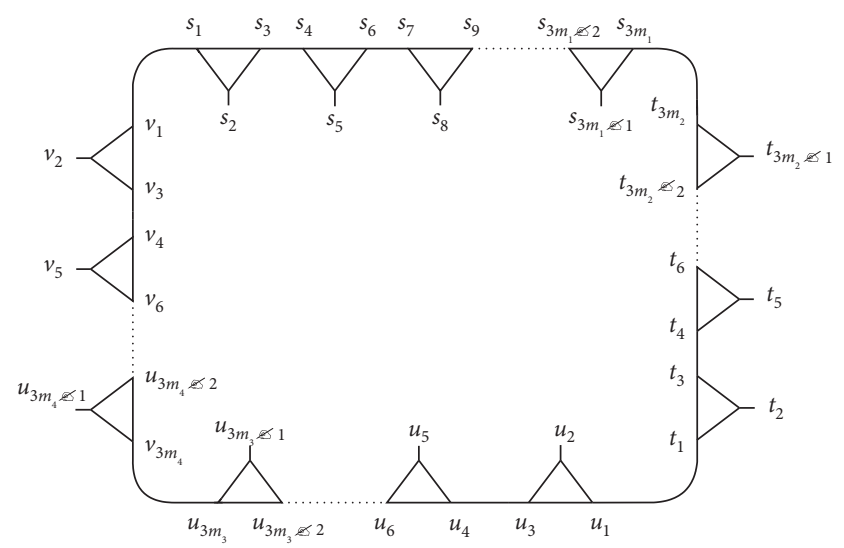

FIgURE 5: The closed path $\left(m_{1}, m_{2}, m_{3}, m_{4}\right)$, where $m_{1}>m_{2}>m_{3}$ and $m_{2}=m_{4}$

contracting $u_{1}^{*}$ and $u_{2}^{*}$. It should be noted that $u_{1}$ and $u_{2}$ do not need to lie in the same closed path $C$. From Figure 5, we have

$$
\begin{aligned}
s_{i}^{*} & =s_{3 m_{1}-(i-1)}, \\
t_{j}^{*} & =v_{3 m_{2}-(j-1)}, \\
u_{k}^{*} & =u_{3 m_{3}-(k-1)}, \\
v_{l}^{*} & =t_{3 m_{4}-(l-1)},
\end{aligned}
$$

for $i=1,2,3, \ldots, 3 m_{1}, j=1,2,3, \ldots, 3 m_{2}, k=1,2,3, \ldots$, $3 m_{3}$, and $l=1,2,3, \ldots, 3 m_{4}$. Thus, for each vertex $u$ in $\psi$, there exists a vertex $u^{*}$ in $\psi$. 
Remark 3. Some homomorphic copies have symmetry about the vertical axis; that is, they have the same orientations as those of their mirror images. In other words, they are mirror images of themselves. The homomorphic copy $\gamma$ of any circuit $C$ having a vertex $u$ fixed by $x_{1}, x_{2}$ has a symmetry about vertical axis if and only if $\gamma$ contains a vertex $u^{*}$ fixed by $x_{1}^{*}, x_{2}^{*}$.

3.1. Proposed Scheme. Since $\psi$ has $3\left(m_{1}+m_{2}+m_{3}+m_{4}\right)$ number of vertices, the total number of pairs in $\psi$ is $\left(\begin{array}{c}3\left(m_{1}+m_{2}+m_{3}+m_{4}\right) \\ 2\end{array}\right)$. We contract a pair of vertices $u_{1}, u_{2}$ of $\psi$ such that a homomorphic copy $\gamma$ is obtained. By using Theorem 2, we find all pairs of vertices in $\psi$, which form $\gamma$; let those be $n$ in numbers. Now, we have two possibilities:

(i) If by contracting $u_{1}$ and $u_{2}$ vertices $u_{1}^{*}$ and $u_{2}^{*}$ are not contracted, then $\gamma$ does not possess a vertical symmetry. Therefore, $\gamma^{*}$ consumes $n$ more pairs of vertices of $\psi$.

(ii) If $u_{1}, u_{2}$ and $u_{1}^{*}, u_{2}^{*}$ are contracted all together, then $\gamma$ has a symmetry about vertical axis. Therefore, $\gamma^{*}$ does not consume any pair. Thus, $\gamma$ has $n$ pairs of vertices.

Next, we contract $\psi$ at one of the remaining pairs and the process continues until all $\left(\begin{array}{c}3\left(m_{1}+m_{2}+m_{3}+m_{4}\right) \\ 2\end{array}\right)$ pairs are exhausted.

Let $i_{1}=0,1,2, \ldots, m_{2}-1$. First, we contract vertex $s_{3 m_{1}}$ with vertices $s_{3 i_{1}+1}$ and the following result is obtained.

Theorem 3. If vertex $s_{3 m_{1}}$ is contracted with vertices $s_{3 i_{1}+1}$, then $m_{2}$ distinct homomorphic copies $\alpha_{i_{1}}$ of $\psi$ are obtained. Furthermore, there are $3\left(m_{2}^{2}+3 m_{2}-2\right)$ pairs of vertices for these homomorphic copies.

Proof. Let $D_{1}=\left\{\alpha_{i_{1}}: i_{1}=0,1,2, \ldots, m_{2}-1\right\}$ be the set of homomorphic copies of $\psi$ obtained by contracting $s_{3 m_{1}}$ with $s_{3 i_{1}+1}$. In Figure 5, it can be seen that $(f g)^{m_{2}}\left(f g^{-1}\right)^{m_{3}}(f g)^{m_{4}}\left(f g^{-1}\right)^{i_{1}} f$ and $g^{-1}\left(f g^{-1}\right)^{m_{1}-i_{1}-1}$ are the possible paths between $s_{3 m_{1}}$ and $s_{3 i_{1}+1}$. Therefore, vertex $s_{3 m_{1}}=s_{3 i_{1}+1}$ in $D_{1}$ is fixed by $(f g)^{m_{2}}\left(f g^{-1}\right)^{m_{3}}(f g)^{m_{4}}\left(f g^{-1}\right)^{i_{1}} f$ and $g^{-1}\left(f g^{-1}\right)^{m_{1}-i_{1}-1}$. It is also clear from Figure 5 that $E_{1}=$ $\left\{f, f g^{-1}, f g, f g f, f g f g^{-1}, \quad(f g)^{2}, \ldots,(f g)^{i_{1}},(f g)^{i_{1}} f\right.$, $\left.(f g)^{i_{1}} f g^{-1},(f g)^{i_{1}+1}, g, g^{-1}, e\right\}$ is the set of elements $x$ of the modular group such that, for all $x \in E_{1}$, both $\left(s_{3 m_{1}}\right) x$ and $\left(s_{3 i_{1}+1}\right) x$ lie in $\psi$. Since $\left|E_{1}\right|=3\left(i_{1}+2\right)$, by Theorem 2 , there are $3\left(i_{1}+2\right)$ pairs in $\psi$ to form $\alpha_{i_{1}}$.

Next, we show that all homomorphic copies $\alpha_{i_{1}}$ of $\psi$ in $D_{1}$ are different and no copy of these is a mirror image of another.

Let $\alpha_{j}, \alpha_{k} \in D_{1}$; then $\alpha_{j}$ is evolved by contracting $s_{3 m_{1}}$ and $s_{3 j+1}$, whereas $\alpha_{k}$ is obtained by contracting $s_{3 m_{1}}$ and $s_{3 k+1}$. Now $\alpha_{j}=\alpha_{k}$ if and only if there exists an element $x$ in $E_{1}$ such that $\left(s_{3 m_{1}}\right) x=s_{3 m_{1}}$ and $\left(s_{3 j+1}\right) x=s_{3 k+1}$. One can see that only $e \in E_{1}$ maps $s_{3 m_{1}}$ to itself, but $\left(s_{3 j+1}\right) e \neq s_{3 k+1}$.
Now suppose that $\alpha_{j}=\alpha_{k}^{*}$; then there must exist some $x$ in $E_{1}$ which sends $s_{3 m_{1}}$ to $s_{3 m_{1}}^{*}=s_{1}$ and $s_{3 j+1}$ to $s_{3 k+1}^{*}=s_{3\left(m_{1}-k\right)}$. But $E_{1}$ does not contain such element. This means that all diagrams in $D_{1}$ are distinct. Thus, $\left|D_{1}\right|=m_{2}$ and there are $3 \sum_{i_{1}=0}^{m_{2}-1}\left(i_{1}+2\right)$ pairs of vertices to create $\alpha_{i_{1}}$.

Now we check how many diagrams in $D_{1}$ have a symmetry about vertical axis. For this, let $\alpha_{j}=\alpha_{j}^{*}$; then $E_{1}$ contains an element $x$ such that

$$
\begin{gathered}
\left(s_{3 m_{1}}\right) x=s_{3 m_{1}}^{*}=s_{1}, \\
\left(s_{3 j+1}\right) x=s_{3 j+1}^{*}=s_{3\left(m_{1}-j\right),} \\
\text { or }\left(s_{3 m_{1}}\right) x=s_{3 j+1}^{*}=s_{3\left(m_{1}-j\right)}, \\
\left(s_{3 j+1}\right) x=s_{3 m_{1}}^{*}=s_{1} .
\end{gathered}
$$

This is possible only if $i_{1}=0$; in this case, we have $e \in E_{1}$ such that $\left(s_{3 m_{1}}\right) e=s_{1}^{*}$ and $\left(s_{1}\right) e=s_{3 m_{1}}^{*}=s_{1}$. So, we conclude that only $\alpha_{0}$ has a symmetry about vertical axis; that is, $\alpha_{0}$ and its mirror image $\alpha_{0}^{*}$ have the same orientations, and all other $m_{2}-1$ homomorphic copies in $\psi$ do not possess a vertical symmetry. Hence there are $6 \sum_{i_{2}=1}^{m_{2}-1}\left(i_{2}+2\right)+6=3\left(m_{2}^{2}+\right.$ $\left.3 m_{2}-2\right)$ pairs to form $D_{1}=\left\{\alpha_{i_{1}}: i_{1}=0,1,2, \ldots, m_{2}-1\right\}$.

We obtain all the results by using the same technique, so, from now onwards, instead of providing proofs of the theorems, we will present tables, which give the necessary information of the family of homomorphic copies evolved.

Let $i_{2}=1,2, \ldots, m_{2}-1$. We contract the pair $t_{3 m_{2}}$ and $t_{3 i_{2}+1}$ and obtain the following result. By using Theorem 1, one can see that these vertices are not contracted in Theorem 3 .

Theorem 4. If vertex $t_{3 m_{2}}$ is contracted with vertices $t_{3 i_{2}+1}$, then $m_{2}-1$ distinct homomorphic copies $\alpha_{i_{2}}^{\prime}$ of $\psi$ are $o b$ tained. Furthermore, there are $3\left(m_{2}^{2}+3 m_{2}-4\right)$ pairs of vertices for these homomorphic copies.

Table 1 shows the complete information of the family of homomorphic copies $\alpha_{i}^{\prime}$. The information provided in Table 1 can be verified by the same technique used in the proof of Theorem 3.

Let us contract $u_{3 m_{3}}$ with $u_{3 i_{3}+1}$, where $i_{3}=0,1,2, \ldots, m_{3}-1$. By using Theorem 1 , it can be easily verified that these pairs of vertices are not utilized in the previous theorems.

Theorem 5. If vertex $u_{3 m_{3}}$ is contracted with vertices $u_{3 i_{3}+1}$, then $m_{3}$ distinct homomorphic copies $\alpha_{i_{3}}^{\prime \prime}$ of $\psi$ are obtained. Furthermore, there are $3\left(m_{3}^{2}+3 m_{3}-2\right)$ pairs of vertices for these homomorphic copies.

All details of the family of homomorphic copies $\alpha_{i_{3}}$ h have been provided in Table 2 .

Next, we contract $s_{3 m_{1}}$ with $t_{3 i_{1}+1}$ and formulate the following Theorem.

Theorem 6. If vertex $s_{3 m_{1}}$ is contracted with vertices $t_{3 i_{1}+1}$, then $m_{2}$ distinct homomorphic copies $\beta_{i_{1}}$ of $\psi$ are obtained. Furthermore, there are $3\left(m_{2}^{2}+3 m_{2}\right)$ pairs of vertices for these homomorphic copies. 
TABLE 1: Necessary information regarding homomorphic copies $\left\{\alpha_{i_{2}}^{\prime}\right\}$.

$\begin{array}{lc}\text { The pairs that are contracted } & \left(t_{3 m_{2}}, t_{3 i_{2}+1}\right) \\ \text { Family of homomorphic copies evolved } & D_{2}=\left\{\alpha_{i_{2}}^{\prime}\right\} \\ \left|D_{2}\right| & m_{2}-1 \\ \text { Number of pairs for each } \alpha_{i_{2}}^{\prime} & 3\left(i_{2}+2\right) \\ \text { Number of homomorphic copies that have } & 0 \\ \text { vertical axis of symmetry } & 3\left(m_{2}^{2}+3 m_{2}-4\right) \\ \text { Total number of pairs for } D_{2}\end{array}$

TABLE 2: Necessary information regarding homomorphic copies $\left\{\alpha_{i_{3}}^{\prime \prime}\right\}$.

The pairs that are contracted

$\left(u_{3 m_{3}}, u_{3 i_{3}+1}\right)$
$D_{3}=\left\{\alpha_{i_{3}}^{\prime \prime}\right\}$
$m_{3}$
$3\left(i_{3}+2\right)$
Only $\alpha_{0}^{\prime \prime}$
$3\left(m_{3}^{2}+3 m_{3}-2\right)$

Family of homomorphic copies evolved

$\left|D_{3}\right|$

Number of pairs for each $\alpha_{i_{3}}^{\prime \prime}$

Homomorphic copies possessing vertical axis

of symmetry

Total number of pairs $D_{3}$

$3\left(m_{3}^{2}+3 m_{3}-2\right)$

For complete information of $\beta_{i}$, see Table 3 .

Now we contract vertex $s_{3 m_{1}}$ with vertices $u_{3 i_{3}+1}$, where $i_{3}=0,1,2, \ldots, m_{3}-1$, and acquire the following result.

Theorem 7. If vertex $s_{3 m_{1}}$ is contracted with vertices $u_{3 i_{3}+1}$, then $m_{3}$ distinct homomorphic copies $\beta_{i_{3}}{ }^{\prime}$ of $\psi$ are obtained. Furthermore, there are $3\left(m_{3}^{2}+3 m_{3}\right)$ pairs of vertices for these homomorphic copies.

Table 4 provides all information regarding homomorphic copies $\beta_{i}{ }^{\prime}$, evolved in Theorem 7 .

The following theorem is evolved by contracting $s_{3 m_{1}}$ with $v_{3 i_{4}+1}$, where $i_{4}=0,1,2, \ldots, m_{4}-1$.

Theorem 8. If vertex $s_{3 m_{1}}$ is contracted with vertices $v_{3 i_{4}+1}$, then $m_{4}$ distinct homomorphic copies $\beta_{i_{4}}^{\prime \prime}$ of $\psi$ are obtained. Furthermore, there are $(3 / 2)\left(m_{4}^{2}+3 m_{4}\right)$ pairs of vertices for these homomorphic copies.

The complete information of the family of homomorphic copies $\beta_{i_{4}}^{\prime \prime}$ obtained in Theorem 8 is given in Table 5 .

The next theorem is obtained by contracting $t_{3 m_{2}}$ with $s_{3 i_{5}+1}$, where $i_{5}=1,2, \ldots, m_{1}-1$.

Theorem 9. If vertex $t_{3 m_{2}}$ is contracted with vertices $s_{3 i_{5}+1}$, then $m_{1}-1$ distinct homomorphic copies $\beta_{i_{5}}^{\prime \prime}$ of $\psi$ are obtained. Furthermore, there are $(3 / 2)\left(m_{1}^{2}+3 m_{1}-4\right)$ pairs of vertices for these homomorphic copies.

All details of the generated homomorphic copies are given in Table 6.

Now we contract vertex $t_{3 m_{2}}$ with vertices $u_{3 i_{6}+1}$ for all $i_{6}=1,2, \ldots, m_{3}-1$.

Theorem 10. If vertex $t_{3 m_{2}}$ is contracted with vertices $u_{3 i_{6}+1}$, then $m_{3}-1$ distinct homomorphic copies $\beta_{i_{6}}^{i v}$ of $\psi$ are obtained. Furthermore, there are $3\left(m_{3}^{2}+3 m_{3}-4\right)$ pairs of vertices for these homomorphic copies.
TABLE 3: Necessary information regarding homomorphic copies $\left\{\beta_{i_{1}}\right\}$.

\begin{tabular}{lc}
\hline The pairs that are contracted & $\left(s_{3 m_{1}}, t_{3 i_{1}+1}\right)$ \\
Family of homomorphic copies evolved & $D_{4}=\left\{\beta_{i_{1}}\right\}$ \\
$\left|D_{4}\right|$ & $m_{2}$ \\
Number of pairs for each $\beta_{i_{1}}$ & $m_{2}$ \\
Homomorphic copies possessing vertical axis of & No one \\
symmetry & $3\left(m_{2}^{2}+3 m_{2}\right)$ \\
Total number of pairs for $D_{4}$
\end{tabular}

TABLE 4: Necessary information regarding homomorphic copies $\left\{\beta_{i_{3}}^{\prime}\right\}$.

\begin{tabular}{lc}
\hline The pairs that are contracted & $\left(s_{3 m_{1}}, u_{3 i_{3}+1}\right)$ \\
Family of homomorphic copies evolved & $D_{5}=\left\{\beta_{i_{3}}^{\prime}\right\}$ \\
$\left|D_{5}\right|$ & $m_{3}$ \\
Number of pairs for each $\beta_{i_{3}}^{\prime}$ & $3\left(i_{3}+2\right)$ \\
Homomorphic copies possessing vertical axis of & No one \\
symmetry & $3\left(m_{3}^{2}+3 m_{3}\right)$ \\
Total number of pairs for $D_{5}$ &
\end{tabular}

TABLE 5: Necessary information regarding homomorphic copies $\left\{\beta_{i_{4}}^{\prime \prime}\right\}$.

\begin{tabular}{lc} 
The pairs that are contracted & $\left(s_{3 m_{1}}, v_{3 i_{4}+1}\right)$ \\
Family of homomorphic copies evolved & $D_{6}=\left\{\beta_{i_{4}}^{\prime \prime}\right\}$ \\
$\left|D_{6}\right|$ & $m_{4}$ \\
Number of pairs for each $\beta_{i_{4}}^{\prime \prime}$ & $3\left(i_{4}+2\right)$ \\
$\begin{array}{l}\text { Homomorphic copies possessing vertical axis } \\
\text { of symmetry }\end{array}$ & All $\beta_{i_{4}}^{\prime \prime}$ \\
Total number of pairs for $D_{6}$ & $(3 / 2)\left(m_{4}^{2}+3 m_{4}\right)$ \\
\hline
\end{tabular}

TABLE 6: Necessary information regarding homomorphic copies $\left\{\beta_{i_{5}}^{\prime \prime}\right\}$.

\begin{tabular}{lc}
\hline The pairs that are contracted & $\left(t_{3 m_{2}}, s_{3 i_{5}+1}\right)$ \\
Family of homomorphic copies evolved & $D_{7}=\left\{\beta_{i_{5}}^{\prime \prime}\right\}$ \\
$\left|D_{7}\right|$ & $m_{1}-1$ \\
Number of pairs for each $\beta_{i_{5}}^{\prime \prime}$ & $3\left(i_{5}+2\right)$ \\
Homomorphic copies possessing vertical & All $\beta_{i_{5}}^{\prime \prime}$ \\
axis of symmetry & $(3 / 2)\left(m_{1}^{2}+3 m_{1}-4\right)$ \\
Total number of pairs for $D_{7}$
\end{tabular}

The complete information of the family of homomorphic copies $\beta_{i_{6}}^{i v}$ is provided in Table 7 .

In the following theorem, $u_{3 m_{3}}$ is contracted with $t_{3 i_{1}+1}$.

Theorem 11. If vertex $u_{3 m_{3}}$ is contracted with vertices $t_{3 i_{1}+1}$, then $m_{2}$ distinct homomorphic copies $\beta_{i_{1}}^{v}$ of $\psi$ are obtained. Furthermore, there are $(3 / 2)\left(m_{2}^{2}+3 m_{2}\right)$ pairs of vertices for these homomorphic copies.

All details of homomorphic copies $\beta_{i_{1}}^{v}$ can be found in Table 8.

Let us now contract vertex $v_{3 m_{4}}$ with vertices $u_{3 i_{6}+1}$, where $i_{6}=1,2, \ldots, m_{3}-1$. 
TABLE 7: Necessary information regarding homomorphic copies $\left\{\beta_{i_{6}}^{i v}\right\}$.

\begin{tabular}{lc}
\hline The pairs that are contracted & $\left(t_{3 m_{2}}, u_{3 i_{6}+1}\right)$ \\
Family of homomorphic copies evolved & $D_{8}=\left\{\beta_{i_{6}}^{i v}\right\}$ \\
$\left|D_{8}\right|$ & $m_{3}-1$ \\
Number of pairs for each $\beta_{i_{6}}^{i v}$ & $3\left(i_{6}+2\right)$ \\
Homomorphic copies possessing vertical axis & No one \\
of symmetry & $3\left(m_{3}^{2}+3 m_{3}-4\right)$ \\
Total number of pairs for $D_{8}$ &
\end{tabular}

TABLE 8: Necessary information regarding homomorphic copies $\left\{\beta_{i_{1}}^{v}\right\}$.

\begin{tabular}{lc}
\hline The pairs that are contracted & $\left(u_{3 m_{3}}, t_{3 i_{1}+1}\right)$ \\
Family of homomorphic copies evolved & $D_{9}=\left\{\beta_{i_{1}}^{v}\right\}$ \\
$\left|D_{9}\right|$ & $m_{2}$ \\
Number of pairs for each $\beta_{i_{1}}^{v}$ & $3\left(i_{1}+2\right)$ \\
Homomorphic copies possessing vertical axis & All $\beta_{i_{1}}^{v}$ \\
of symmetry & $(3 / 2)\left(m_{2}^{2}+3 m_{2}\right)$ \\
Total number of pairs for $D_{9}$
\end{tabular}

TABLE 9: Necessary information regarding homomorphic copies $\left\{\beta_{i_{6}}^{v i}\right\}$.

The pairs that are contracted

$$
\begin{gathered}
\left(v_{3 m_{4}}, u_{3 i_{6}+1}\right) \\
D_{10}=\left\{\beta_{i_{6}}^{v i}\right\} \\
m_{3}-1 \\
3\left(i_{6}+2\right)
\end{gathered}
$$

Family of homomorphic copies evolved $\left|D_{10}\right|$ Number of pairs for each $\beta_{i_{6}}^{v i}$

Homomorphic copies possessing vertical axis of symmetry

Total number of pairs for $D_{10}$

All $\beta_{i_{6}}^{v i}$

$(3 / 2)\left(m_{3}^{2}+3 m_{3}-4\right)$

Theorem 12. If vertex $v_{3 m_{4}}$ is contracted with vertices $u_{3 i_{6}+1}$, then $m_{3}-1$ distinct homomorphic copies $\beta_{i_{6}}^{v i}$ of $\psi$ are obtained. Furthermore, there are $(3 / 2)\left(m_{3}^{2}+3 m_{3}-4\right)$ pairs of vertices for these homomorphic copies.

All information related to the homomorphic copies created in Theorem 12 is given in Table 9.

Let us contract vertex $s_{3 m_{1}}$ with vertices $s_{3 i_{7}+1}$, where $i_{7}=m_{2}+1, m_{2}+2, \ldots, m_{1}-1$. Consequently, we have the following theorem.

Theorem 13. If vertex $s_{3 m_{1}}$ is contracted with vertices $s_{3 i_{7}+1}$, then $m_{1}-m_{2}-1$ distinct homomorphic copies $\gamma_{i_{7}}$ of $\psi$ are obtained. Furthermore, there are $6\left(m_{2}+2\right)\left(m_{1}-m_{2}-1\right)$ pairs of vertices for these homomorphic copies.

Table 10 completely describes the family of homomorphic copies evolved in Theorem 11.

Suppose that $\varepsilon_{1}= \begin{cases}0, & \text { if } m_{1}+m_{3} \text { is even } \\ 1, & \text { if } m_{1}+m_{3} \text { is odd }\end{cases}$ and $i_{8}=m_{3}+1, m_{3}+2, \ldots,\left(\left(m_{1}+m_{3}-\varepsilon_{1}\right) / 2\right)$. Let us contract $v_{3 m_{4}}$ with $s_{3 i_{8}+1}$ to obtain Theorem 14 .

Theorem 14. If vertex $v_{3 m_{4}}$ is contracted with vertices $s_{3 i_{8}+1}$, then $(1 / 2)\left(m_{1}-m_{3}-\varepsilon_{1}\right)$ distinct homomorphic copies $\gamma_{i_{8}}^{\prime}$ of $\psi$
TABLE 10: Necessary information regarding homomorphic copies $\left\{\gamma_{i_{7}}\right\}$.

\begin{tabular}{lc}
\hline The pairs that are contracted & $\left(s_{3 m_{1}}, s_{3 i_{7}+1}\right)$ \\
Family of homomorphic copies evolved & $D_{11}=\left\{\gamma_{i_{7}}\right\}$ \\
$\left|D_{11}\right|$ & $m_{1}-m_{2}-1$ \\
Number of pairs for each $\gamma_{i_{7}}$ & $3\left(m_{2}+2\right)$ \\
Homomorphic copies possessing & No one \\
vertical axis of symmetry & $6\left(m_{2}+2\right)\left(m_{1}-m_{2}-1\right)$ \\
Total number of pairs for $D_{11}$ &
\end{tabular}

TABLE 11: Necessary information regarding homomorphic copies $\left\{\gamma_{i_{8}}^{\prime}\right\}$.

The pairs that are contracted

Family of homomorphic copies evolved

$\left|D_{12}\right|$

Number of pairs for each $\gamma_{i_{8}}^{\prime}$

Homomorphic copies possessing

vertical axis of symmetry

$$
\begin{aligned}
& \left(v_{3 m_{4}}, s_{3 i_{8}+1}\right) \\
& D_{12}=\left\{\gamma_{i_{8}}^{\prime}\right\}
\end{aligned}
$$

$$
(1 / 2)\left(m_{1}-m_{3}-\varepsilon_{1}\right)
$$$$
3\left(m_{3}+2\right)
$$

No one if $m_{1}+m_{3}$ is odd and

$\gamma_{\left(\left(m_{1}+m_{3}\right) / 2\right)}$ if $m_{1}+m_{3}$ is

Total number of pairs for $D_{12}$

$3\left(m_{3}+2\right)\left(m_{1}-m_{3}-1\right)$

are obtained. Furthermore, there are $3\left(m_{3}+2\right)\left(m_{1}-m_{3}-1\right)$ pairs of vertices for these homomorphic copies.

All information of the homomorphic copies evolved in Theorem 14 is provided in Table 11 .

The following theorem emerges as a result of contracting $v_{3 m_{4}}$ with $t_{3 i_{9}+1}$, where $i_{9}=m_{3}+1, m_{3}+2, \ldots, m_{2}-1$.

Theorem 15. If vertex $v_{3 m_{4}}$ is contracted with vertices $t_{3 i_{9}+1}$, then $m_{2}-m_{3}-1$ distinct homomorphic copies $\gamma_{i_{9}}^{\prime \prime}$ of $\psi$ are obtained. Furthermore, there are $6\left(m_{3}+2\right)\left(m_{2}-m_{3}-1\right)$ pairs of vertices for these homomorphic copies.

Table 12 shows the complete information of all homomorphic copies created in Theorem 15.

Suppose that $i_{10}=1,2, \ldots, m_{1}-1$ and $i_{11}=1,2, \ldots$, $m_{2}-1$. Let us contract $s_{3 i_{10}+1}$ with $t_{3 i_{11}}$ and obtain the following results.

Theorem 16. If vertices $s_{3 i_{10}+1}$ are contracted with vertices $t_{3 i_{11}}$, then $\left(m_{1}-1\right)\left(m_{2}-1\right)$ distinct homomorphic copies $\delta_{\left(i_{10}, i_{11}\right)}$ of $\psi$ are obtained. Furthermore, there are $12\left(m_{1}-\right.$ 1) $\left(m_{2}-1\right)$ pairs of vertices for these homomorphic copies.

All necessary details of the family of homomorphic copies evolved in this process are provided in Table 13.

Let $\quad i_{12}=1,2,3, \ldots,\left(\left(m_{3}-\varepsilon_{2}-2\right) / 2\right), \quad$ where $\varepsilon_{2}=\left\{\begin{array}{ll}0, & \text { if } m_{3} \text { is even } \\ 1, & \text { if } m_{3} \text { is odd }\end{array}\right.$. The following results have been constructed by contracting $s_{3 i_{10}+1}$ with $u_{3 i_{12}}$.

Theorem 17. If vertices $s_{3 i_{10}+1}$ are contracted with vertices $u_{3 i_{12}}$, then $(1 / 2)\left(m_{1}-1\right)\left(m_{3}-\varepsilon_{2}-2\right)$ distinct homomorphic 
TABLE 12: Necessary information regarding homomorphic copies $\left\{\gamma_{i_{9}}^{\prime \prime}\right\}$.

$\begin{array}{lc}\text { The pairs that are contracted } & \left(v_{3 m_{4}}, t_{3 i_{9}+1}\right) \\ \text { Family of homomorphic copies evolved } & D_{13}=\left\{\gamma_{i_{9}}^{\prime \prime}\right\} \\ \left|D_{13}\right| & m_{2}-m_{3}-1 \\ \text { Number of pairs for each } \gamma_{i_{9}}^{\prime \prime} & 3\left(m_{3}+2\right) \\ \text { Homomorphic copies possessing } & \text { No one } \\ \text { vertical axis of symmetry } & 6\left(m_{3}+2\right)\left(m_{2}-m_{3}-1\right) \\ \text { Total number of pairs for } D_{13} & \end{array}$

TABLE 13: Necessary information regarding homomorphic copies $\left\{\delta_{\left(i_{10}, i_{11}\right)}\right\}$.

The pairs that are contracted

$$
\begin{gathered}
\left(s_{3 i_{10}+1}, t_{3 i_{11}}\right) \\
D_{14}=\left\{\delta_{\left(i_{10}, i_{11}\right.}\right\} \\
\left(m_{1}-1\right)\left(m_{2}-1\right) \\
6
\end{gathered}
$$

Family of homomorphic copies evolved

$\left|D_{14}\right|$

Number of pairs for each $\delta_{\left(i_{10}, i_{11}\right)}$

Homomorphic copies possessing vertical axis of symmetry

Total number of pairs for $D_{14}$

No one

$12\left(m_{1}-1\right)\left(m_{2}-1\right)$

TABLE 14: Necessary information regarding homomorphic copies $\left\{\delta_{\left(i_{10}, i_{12}\right)}^{\prime}\right\}$.
The pairs that are contracted

Family of homomorphic copies evolved

$\left|D_{15}\right|$

Number of pairs for each $\delta_{\left(i_{10}, i_{12}\right)}$

Homomorphic copies possessing

vertical axis of symmetry

Total number of pairs for $D_{15}$

$$
\begin{gathered}
\left(s_{3 i_{10}+1}, u_{3 i_{12}}\right) \\
D_{15}=\left\{\delta_{\left(i_{10}, i_{12}\right)}{ }^{\prime}\right\} \\
(1 / 2)\left(m_{1}-1\right)\left(m_{3}-\varepsilon_{2}-2\right) \\
6
\end{gathered}
$$

No one $6\left(m_{1}-1\right)\left(m_{3}-\varepsilon_{2}-2\right)$ copies $\delta_{\left(i_{10}, i_{12}\right)}^{\prime}$ of $\psi$ are obtained. Furthermore, there are $6\left(m_{1}-1\right)\left(m_{3}-\varepsilon_{2}-2\right)$ pairs of vertices for these homomorphic copies.

See Table 14 for complete information of the homomorphic copies.

Recall that $i_{13}=1,2, \ldots, m_{1}-1 \quad$ and $\varepsilon_{2}=\left\{\begin{array}{ll}0 & \text { if } m_{3} \text { is even } \\ 1 & \text { if } m_{3} \text { is odd }\end{array}\right.$. Let $\varepsilon_{3}=\left\{\begin{array}{ll}0 & \text { if } m_{1} \text { is even } \\ 1 & \text { if } m_{1} \text { is odd }\end{array}\right.$, and we obtain the following theorem by contracting $s_{3 i_{13}+1}$ with $u_{3\left(\left(m_{3}-\varepsilon_{2}\right) / 2\right)}$.

Theorem 18. If vertices $s_{3 i_{13}+1}$ are contracted with vertex $u_{3\left(\left(m_{3}-\varepsilon_{2}\right) / 2\right)}$ then $\left\{\begin{array}{ll}\left(\left(m_{1}-\varepsilon_{2}\right) / 2\right) & \text { if } m_{3} \text { is even } \\ m_{1}-1 & \text { if } m_{3} \text { is odd }\end{array}\right.$ distinct homomorphic copies $\delta_{\left(i_{13},\left(\left(m_{3}-i_{3}\right) / 2\right)\right)}$ of $\psi$ are obtained. Fur-

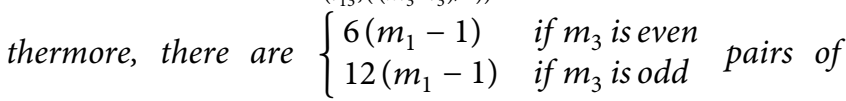
vertices for these homomorphic copies.

All information related to the homomorphic copies created in Theorem 18 is provided in Table 15.
Now we contract $t_{3 i_{14}+1}$ with $u_{3 i_{15}}$, where $i_{14}=1,2, \ldots, m_{2}-1$ and $i_{15}=1,2, \ldots, m_{3}-1$, so that Theorem 19 is evolved.

Theorem 19. If vertices $t_{3 i_{14}+1}$ are contracted with vertices $u_{3 i_{15}}$, then $\left(m_{2}-1\right)\left(m_{3}-1\right)$ distinct homomorphic copies $\delta_{\left(i_{14}, i_{15}\right)}$ of $\psi$ are obtained. Furthermore, there are $12\left(m_{2}-\right.$ 1) $\left(m_{3}-1\right)$ pairs of vertices for these homomorphic copies.

For complete details about the homomorphic copies evolved in Theorem 19, see Table 16.

Let $i_{16}=1,2,3, \ldots, m_{4}-i_{14}$. The following theorem is obtained by contracting $t_{3 i_{14}+1}$ with $v_{3 i_{16}}$.

Theorem 20. If vertices $t_{3 i_{14}+1}$ are contracted with vertices $v_{3 i_{16}}$, then $(1 / 2) m_{2}\left(m_{2}-1\right)$ distinct homomorphic copies $\delta_{\left(i_{14}, i_{16}\right)}^{i{ }^{16}}$ of $\psi$ are obtained. Furthermore, there are $6\left(m_{2}-1\right)^{2}$ pairs of vertices for these homomorphic copies.

All important information regarding $\delta_{\left(i_{14}, i_{16}\right)}^{i v}$ is provided in Table 17.

Recall that $\varepsilon_{3}=\left\{\begin{array}{ll}0 & \text { if } m_{1} \text { is even } \\ 1 & \text { if } m_{1} \text { is odd }\end{array}\right.$, and let $i_{17}=1,2,3, \ldots,\left(\left(m_{1}-2-\varepsilon_{3}\right) / 2\right) \quad$ and $i_{18}=i_{17}+1$, $i_{17}+2, \ldots, m_{1}-i_{17}$. In Theorem 21, we contract vertices $s_{3 i_{17}+1}$ with vertices $s_{3 i_{18}}$ to create homomorphic copies $\mu_{\left(i_{17}, i_{18}\right)}$.

Theorem 21. If vertices $s_{3 i_{17}+1}$ are contracted with vertices $s_{3 i_{18}}$, then $(1 / 4)\left(m_{1}^{2}-2 m_{1}+\varepsilon_{3}\right)$ distinct homomorphic copies $\mu_{\left(i_{17}, i_{18}\right)}$ of $\psi$ are obtained. Furthermore, there are $3\left(m_{1}^{2}-\right.$ $\left.3 m_{1}+2\right)$ pairs of vertices for these homomorphic copies.

All information about the family of homomorphic copies evolved in Theorem 21 is given in Table 18 .

Now, let us contract vertices $t_{3 i_{19}+1}$ with $t_{3 i_{20}}$, where $i_{19}=$ $1,2,3, \ldots, m_{2}-2$ and $i_{20}=i_{19}+1, i_{19}+2, i_{19}+3$, $\ldots, m_{2}-1$.

Theorem 22. If vertices $t_{3 i_{19}+1}$ are contracted with vertices $t_{3 i_{20}}$, then $(1 / 2)\left(m_{2}-2\right)\left(m_{2}-1\right)$ distinct homomorphic copies $\mu_{\left(i_{1}, i_{20}\right)}$ of $\psi$ are obtained. Furthermore, there are $6\left(m_{2}-2\right)\left(m_{2}-1\right)$ pairs of vertices for these homomorphic copies.

Table 19 provides all information regarding $\mu_{\left(i_{19}, i_{20}\right)}^{\prime}$.

Recall that $\varepsilon_{2}=\left\{\begin{array}{ll}0 & \text { if } m_{3} \text { is even } \\ 1 & \text { if } m_{3} \text { is odd }\end{array}\right.$ and let $i_{21}=$ $1,2,3, \ldots,\left(\left(m_{3}-2+\varepsilon_{2}\right) / 2\right)$ and $i_{22}=i_{21}+1, i_{21}+2$, $i_{21}+3, \ldots, m_{3}-i_{21}$. The next theorem is obtained by contracting $u_{3 i_{21}+1}$ with $u_{3 i_{22}}$

Theorem 23. If vertices $u_{3 i_{21}+1}$ are contracted with vertices $u_{3 i_{22}}$, then $(1 / 4)\left(m_{3}^{2}-2 m_{3}+\varepsilon_{2}\right)$ distinct homomorphic copies $\mu_{\left(i_{21}, i_{22}\right)}$ of $\psi$ are obtained. Furthermore, there are $3\left(m_{3}^{2}-\right.$ $\left.3 m_{3}+2\right)$ pairs of vertices for these homomorphic copies. 
TABLE 15: Necessary information regarding homomorphic copies $\left\{\delta_{\left(i_{13},\left(\left(m_{3}-i_{3}\right) / 2\right)\right)}^{\prime \prime}\right\}$.

The pairs that are contracted

$$
\left(s_{3 i_{13}+1}, u_{3\left(\left(m_{3}-\varepsilon_{2}\right) / 2\right)}\right)
$$

Family of homomorphic copies evolved

$\left|D_{16}\right|$

Number of pairs for each

$\delta_{\left(i_{13},\left(\left(m_{3}-i_{3}\right) / 2\right)\right)}^{\prime \prime}$

Homomorphic copies

possessing vertical axis of $\delta_{\left(\left(m_{1} / 2\right),\left(m_{3} / 2\right)\right)}^{\prime \prime}$ if $m_{1}$ and $m_{3}$ are even symmetry

Total number of pairs for and no one otherwise

$D_{16}$

$$
\begin{cases}6\left(m_{1}-1\right) & \text { if } m_{3} \text { is even } \\ 12\left(m_{1}-1\right) & \text { if } m_{3} \text { is odd }\end{cases}
$$

TABLE 16: Necessary information regarding homomorphic copies $\left\{\delta_{\left(i_{14}, i_{15}\right)}^{\prime \prime}\right\}$.

The pairs that are contracted

$$
\left(t_{3 i_{14}+1}, u_{3 i_{15}}\right)
$$

Family of homomorphic copies evolved

$\left|D_{17}\right|$

Number of pairs for each $\delta_{\left(i_{14}, i_{15}\right)}^{\prime \prime}$

Homomorphic copies possessing vertical axis of symmetry

Total number of pairs for $D_{17}$

$$
\begin{gathered}
D_{17}=\left\{\delta_{\left(i_{14}, i_{15}\right)}^{\prime \prime}\right\} \\
\left(m_{2}-1\right)\left(m_{3}-1\right)
\end{gathered}
$$$$
6
$$

No one

$12\left(m_{2}-1\right)\left(m_{3}-1\right)$

TABLE 17: Necessary information regarding homomorphic copies $\left\{\delta_{\left(i_{14}, i_{16}\right)}^{i v}\right\}$.

The pairs that are contracted

Family of homomorphic copies evolved

$\left|D_{18}\right|$

Number of pairs for each $\delta_{\left(i_{14}, i_{16}\right)}^{i v}$

Homomorphic copies possessing vertical axis

of symmetry

Total number of pairs for $D_{18}$

TABLE 18: Necessary information regarding homomorphic copies $\left\{\mu_{\left(i_{17}, i_{18}\right)}\right\}$.

The pairs that are contracted

Family of homomorphic copies evolved

$\left|D_{19}\right|$

Number of pairs for each $\mu_{\left(i_{17}, i_{18}\right)}$

Homomorphic copies possessing vertical

axis of symmetry

Total number of pairs for $D_{19}$

TABLE 19: Necessary information regarding homomorphic copies $\left\{\mu_{\left(i_{19}, i_{20}\right)}\right\}$.

\begin{tabular}{lc}
\hline The pairs that are contracted & $\left(t_{3 i_{19}+1}, t_{3 i_{20}}\right)$ \\
Family of homomorphic copies evolved & $D_{20}=\left\{\mu_{\left(i_{19}, i_{20}\right)}^{\prime}\right\}$ \\
$\left|D_{20}\right|$ & $(1 / 2)\left(m_{2}-2\right)\left(m_{2}-1\right)$ \\
Number of pairs for each $\mu_{\left(i_{19}, i_{20}\right)}^{\prime}$ & 6 \\
$\begin{array}{l}\text { Homomorphic copies possessing vertical } \\
\text { axis of symmetry }\end{array}$ & No one \\
Total number of pairs for $D_{20}$ & $6\left(m_{2}-2\right)\left(m_{2}-1\right)$ \\
\hline
\end{tabular}

All details of the generated homomorphic copies are given in Table 20.

Now suppose that $i_{23}=1,2,3, \ldots,\left(\left(m_{1}-\varepsilon_{3}\right) / 2\right)$. The following results have been constructed by contracting $s_{3 i_{23}+1}$ with $s_{3 i_{23}}$.

Theorem 24. If vertices $s_{3 i_{23}+1}$ are contracted with vertices $s_{3 i_{23}}$, then $(1 / 2)\left(m_{1}-\varepsilon_{3}\right)$ distinct homomorphic copies $v_{\left(i_{23}, i_{23}\right)}$ of $\psi$ are obtained. Furthermore, there are $3\left(m_{1}-1\right)$ pairs of vertices for these homomorphic copies.

See Table 21 for complete details of the evolved homomorphic copies.

Now, we contract $t_{3 i_{24}+1}$ with $t_{3 i_{24}}$, where $i_{24}=1,2,3, \ldots, m_{2}-1$.

Theorem 25. If vertices $t_{3 i_{24}+1}$ are contracted with vertices $t_{3 i_{24}}$, then $m_{2}-1$ distinct homomorphic copies $\nu_{\left(i_{24}, i_{24}\right)}$ of $\psi$ are obtained. Furthermore, there are $6\left(m_{2}-1\right)$ pairs of vertices for these homomorphic copies.

The complete information of the family of homomorphic copies $\nu_{\left(i_{24}, i_{24}\right)}$ obtained in Theorem 25 is given in Table 22 .

Suppose that $i_{25}=1,2,3, \ldots,\left(\left(m_{3}-\varepsilon_{2}\right) / 2\right)$. Let us contract $u_{3 i_{25}+1}$ and $u_{3 i_{25}}$ and obtain the following results.

Theorem 26. If vertices $u_{3 i_{25}+1}$ are contracted with vertices $u_{3 i_{25}}$, then $(1 / 2)\left(m_{3}-\varepsilon_{2}\right)$ distinct homomorphic copies $v_{\left(i_{25}, i_{25}\right)}^{\prime \prime 25}$ of $\psi$ are evolved. Furthermore, there are $3\left(m_{3}-1\right)$ pairs of vertices for these homomorphic copies.

All information of the family of homomorphic copies $\nu_{\left(i_{25}, i_{25}\right)}^{\prime \prime}$ evolved in Theorem 26 is provided in Table 23.

Lastly, we contract 4 pairs of vertices to evolve 4 homomorphic copies.

\section{Theorem 27}

(i) Let $\eta_{1}$ be the homomorphic copy of $\psi$ obtained by contracting $s_{1}$ and $t_{1}$. Then there are $6\left(m_{2}+m_{3}+\right.$ 2) pairs of vertices for $\eta_{1}$.

(ii) Let $\eta_{2}$ be the homomorphic copy obtained by contracting $s_{1}$ and $u_{1}$. Then there are $3\left(m_{2}+2 m_{3}+2\right)$ pairs of vertices for $\eta_{2}$.

(iii)) Let $\eta_{3}$ be the homomorphic copy obtained by contracting $s_{1}$ and $v_{1}$. Then there are $6\left(m_{2}+1\right)$ pairs of vertices to generate $\eta_{3}$.

(iv) Let $\eta_{4}$ be the homomorphic copy obtained by contracting $t_{1}$ and $u_{1}$. Then there are $6\left(m_{3}+1\right)$ pairs of vertices for $\eta_{4}$.

Let $\Gamma=\left\{\begin{array}{ll}0 & \text { if } m_{1} \text { and } m_{3} \text { are odd } \\ 4 & \text { if } m_{1} \text { and } m_{3} \text { are even } \\ -1 & \text { if } m_{1} \text { is even and } m_{3} \text { is odd or vice versa }\end{array}\right.$.

We are now in a position to prove the main theorem of this paper. 
TABLE 20: Necessary information regarding homomorphic copies $\left\{\mu_{\left(i_{21}, i_{22}\right)}^{\prime \prime}\right\}$.

The pairs that are contracted

Family of homomorphic copies evolved

$\left|D_{21}\right|$

Number of pairs for each $\mu_{\left(i_{2}, i_{22}\right)}^{\prime \prime}$

Homomorphic copies possessing vertical axis of symmetry

Total number of pairs for $D_{21}$

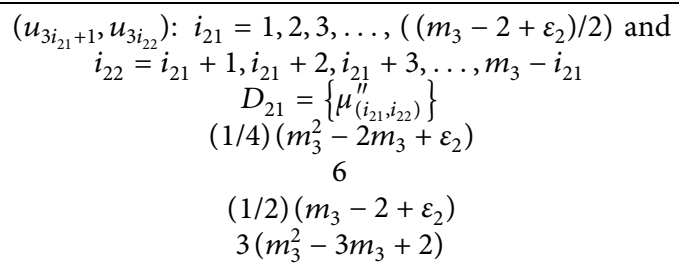

TABLE 21: Necessary information regarding homomorphic copies $\left\{v_{\left(i_{23}, i_{23}\right)}\right\}$.

The pairs that are contracted

Family of homomorphic copies evolved

$\left|D_{22}\right|$

Number of pairs for each $v_{\left(i_{23}, i_{23}\right)}$

Homomorphic copies possessing vertical axis of symmetry

Total number of pairs for $D_{22}$

$$
\begin{gathered}
\left(s_{3 i_{23}+1}, s_{3 i_{23}}\right) \\
D_{22}=\left\{v_{\left(i_{23}, i_{23}\right)}\right\} \\
(1 / 2)\left(m_{1}-\varepsilon_{3}\right) \\
3 \\
v_{\left(\left(m_{1} / 2\right),\left(m_{1} / 2\right)\right)} \\
3\left(m_{1}-1\right)
\end{gathered}
$$

TABLE 22: Necessary information regarding homomorphic copies $\left\{v_{\left(i_{24}, i_{24}\right)}^{\prime}\right\}$.

The pairs that are contracted

Family of homomorphic copies evolved

$\left|D_{23}\right|$

Number of pairs for each $v_{\left(i_{24}, i_{24}\right)}^{\prime}$

Homomorphic copies possessing vertical axis of symmetry

Total number of pairs for $D_{23}$

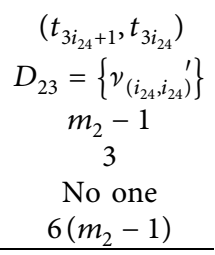

TABLE 23: Necessary information regarding homomorphic copies $\left\{v_{\left(i_{25}, i_{25}\right)}^{\prime \prime}\right\}$.

The pairs that are contracted

$$
\begin{gathered}
\left(u_{3 i_{25}+1}, u_{3 i_{25}}\right) \\
D_{24}=\left\{v_{\left(i_{25}, i_{25}\right)}^{\prime \prime}\right\} \\
(1 / 2)\left(m_{3}-\varepsilon_{2}\right) \\
3 \\
v_{\left(\left(m_{3} / 2\right), m_{3} / 2\right)}^{\prime \prime} \\
3\left(m_{3}-1\right)
\end{gathered}
$$$$
\left|D_{24}\right|
$$$$
\text { Number of pairs for each } \nu_{\left(i_{25}, i_{25}\right)}^{\prime \prime}
$$

Homomorphic copies possessing vertical axis of symmetry

Total number of pairs for $D_{24}$

Theorem 28. There are (1/4) $\left\{m_{1}^{2}+4 m_{2}^{2}+m_{3}^{2}+4\left(m_{1} m_{2}+\right.\right.$ $\left.\left.m_{2} m_{3}\right)+2 m_{1} m_{2}+8 m_{2}+4 m_{3}+\Gamma\right\}$ numbers of distinct homomorphic copies of $\psi$ by contracting all the pairs in $\psi$.

Proof. Let us contract the following pairs of vertices:
(i) $s_{3 m_{1}}$ and $s_{3 i_{1}+1}$
(ii) $t_{3 m_{2}}$ and $t_{3 i_{2}+1}$
(iii) $u_{3 m_{3}}$ and $u_{3 i_{3}+1}$
(iv) $s_{3 m_{1}}$ and $t_{3 i_{1}+1}$
(v) $s_{3 m_{1}}$ and $u_{3 i_{3}+1}$
(vi) $s_{3 m_{1}}$ and $v_{3 i_{4}+1}$
(vii) $t_{3 m_{2}}$ and $s_{3 i_{5}+1}$
(viii) $t_{3 m_{2}}$ and $u_{3 i_{6}+1}$
(ix) $u_{3 m_{3}}$ and $t_{3 i_{1}+1}$
(x) $v_{3 m_{4}}$ and $u_{3 i_{6}+1}$
(xi) $s_{3 m_{1}}$ and $s_{3 i_{7}+1}$
(xii) $v_{3 m_{4}}$ and $s_{3 i_{8}+1}$

(xiii) $v_{3 m_{4}}$ and $t_{3 i_{9}+1}$

(xiv) $s_{3 i_{10}+1}$ and $t_{3 i_{11}}$

(xv) $s_{3 i_{10}+1}$ and $u_{3 i_{12}}$

(xvi) $s_{3 i_{13}+1}$ and $u_{3\left(\left(m_{3}-\varepsilon_{2}\right) / 2\right)}$

(xvii) $t_{3 i_{14}+1}$ and $u_{3 i_{15}}$

(xviii) $t_{3 i_{14}+1}$ and $v_{3 i_{16}}$

(xix) $s_{3 i_{17}+1}$ and $s_{3 i_{18}}$

(xx) $t_{3 i_{19}+1}$ and $t_{3 i_{20}}$

(xxi) $u_{3 i_{21}+1}$ and $u_{3 i_{22}}$

(xxii) $s_{3 i_{23}+1}$ and $s_{3 i_{23}}$

(xxiii) $t_{3 i_{24}+1}$ and $t_{3 i_{24}}$

(xxiv) $u_{3 i_{25}+1}$ and $u_{3 i_{25}}$

(xxv) $s_{1}$ and $t_{1}$

(xxvi) $s_{1}$ and $u_{1}$

(xxvii) $s_{1}$ and $v_{1}$

(xxviii) $t_{1}$ and $u_{1}$

Then, by Theorems 3 to 27 , we obtain the set 


$$
F=\left\{\begin{array}{c}
\alpha_{i_{1}}, \alpha_{i_{2}}^{\prime}, \alpha_{i_{3}}^{\prime \prime}, \beta_{i_{1}}, \beta_{i_{3}}^{\prime}, \beta_{i_{4}}^{\prime \prime}, \beta_{i_{5}}^{\prime \prime}, \beta_{i_{6}}^{i v}, \beta_{i_{1}}^{v}, \beta_{i_{6}}^{v i}, \\
\gamma_{i_{7}}, \gamma_{i_{8}}^{\prime}, \gamma_{i_{9}}^{\prime \prime}, \delta_{\left(i_{10}, i_{11}\right)}, \delta_{\left(i_{10}, i_{12}\right)}^{\prime}, \delta_{\left(i_{13},\left(\left(m_{3}-i_{3}\right) / 2\right)\right)}^{\prime \prime}, \\
\delta_{\left(i_{14}, i_{15}\right)}^{\prime \prime}, \delta_{\left(i_{14}, i_{16}\right)}^{i v}, \mu_{\left(i_{17}, i_{18}\right)}, \mu_{\left(i_{19}, i_{20}\right)}^{\prime}, \mu_{\left(i_{21}, i_{22}\right)}^{\prime \prime}, \nu_{\left(i_{23}, i_{23}\right)}, \nu_{\left(i_{24}, i_{24}\right)}^{\prime}, \nu_{\left(i_{25}, i_{25}\right)}^{\prime \prime}, \\
\eta_{1}, \eta_{2}, \eta_{3}, \eta_{4}
\end{array}\right\},
$$

of homomorphic copies of $\psi$ and there are

$$
\begin{aligned}
S= & 3\left(m_{2}^{2}+3 m_{2}-2\right)+3\left(m_{2}^{2}+3 m_{2}-4\right)+3\left(m_{3}^{2}+3 m_{3}-2\right)+3\left(m_{2}^{2}+3 m_{2}\right) \\
& +3\left(m_{3}^{2}+3 m_{3}\right)+\frac{3}{2}\left(m_{4}^{2}+3 m_{4}\right)+\frac{3}{2}\left(m_{1}^{2}+3 m_{1}-4\right)+3\left(m_{3}^{2}+3 m_{3}-4\right) \\
& +\frac{3}{2}\left(m_{2}^{2}+3 m_{2}\right)+\frac{3}{2}\left(m_{3}^{2}+3 m_{3}-4\right)+6\left(m_{2}+2\right)\left(m_{1}-m_{2}-1\right) \\
& +3\left(m_{3}+2\right)\left(m_{1}-m_{3}-1\right)+6\left(m_{3}+2\right)\left(m_{2}-m_{3}-1\right) \\
& +12\left(m_{1}-1\right)\left(m_{2}-1\right)+6\left(m_{1}-1\right)\left(m_{3}-\varepsilon_{2}-2\right) \\
& +\left\{6\left(m_{1}-1\right) \quad \text { if } m_{3}\right. \text { is even } \\
& +3\left(m_{1}^{2}+3 m_{1}-2\right)+6\left(m_{2}-2\right)\left(m_{2}-1\right)+3\left(m_{3}^{2}-3 m_{3}-2\right) \\
& +3\left(m_{1}-1\right)+6\left(m_{3}-1\right)+3\left(m_{3}-1\right)+6\left(m_{2}+m_{3}+2\right) \\
& +3\left(m_{2}+2 m_{3}+2\right)+6\left(m_{2}+1\right)+6\left(m_{3}-1\right),
\end{aligned}
$$

pairs to form $F . S=\left(\begin{array}{c}3\left(m_{1}+m_{2}+m_{3}+m_{4}\right) \\ 2\end{array}\right)$ is the total number of pairs in $\psi$ and

$$
\begin{aligned}
|F|= & \frac{1}{4}\left\{m_{1}^{2}+4 m_{2}^{2}+m_{3}^{2}+4\left(m_{1} m_{2}+m_{2} m_{3}\right)\right. \\
& \left.+2 m_{1} m_{2}+8 m_{2}+4 m_{3}+\Gamma\right\} .
\end{aligned}
$$

Thus, there are

$$
\frac{1}{4}\left\{m_{1}^{2}+4 m_{2}^{2}+m_{3}^{2}+4\left(m_{1} m_{2}+m_{2} m_{3}\right)+2 m_{1} m_{2}+8 m_{2}+4 m_{3}+\Gamma\right\},
$$

numbers of distinct homomorphic copies obtained by contracting all pairs in $\psi$.

\section{Conclusion}

In this paper, we have highlighted the significance of homomorphic copies of coset graphs. We show how the bigger coset graphs get contracted to transform into their homomorphic copies. As a sample, we consider the closed path $\psi$ of rank 4 ; that is, $\psi=\left(m_{1}, m_{2}, m_{3}, m_{4}\right)$, where $m_{1}>m_{2}>m_{3}$ and $m_{2}=m_{4}$, in coset graphs. We prove that the total numbers of homomorphic copies of $\psi$ are

$$
\begin{aligned}
& \frac{1}{4}\left\{m_{1}^{2}+4 m_{2}^{2}+m_{3}^{2}+4\left(m_{1} m_{2}+m_{2} m_{3}\right)\right. \\
& \left.+2 m_{1} m_{2}+8 m_{2}+4 m_{3}+\Gamma\right\} .
\end{aligned}
$$

It is hoped that the ideas presented in this study will also help to generate the homomorphic copies of other types of graphs for various purposes.

\section{Data Availability}

No data were used to support this study.

\section{Conflicts of Interest}

The authors declare no conflicts of interest regarding the publication of this article.

\section{Acknowledgments}

This paper was supported by Researchers Supporting Project (no. RSP-2021/317), King Saud University, Riyadh, Saudi Arabia. 


\section{References}

[1] M. Akbas, "On suborbital graphs for the modular group," Bulletin of the London Mathematical Society, vol. 6, no. 33, pp. 647-652, 2001.

[2] E. Fujikawa, "Modular groups acting on infinite dimensional Teichmüller spaces," In the Tradition of Ahlfors and Bers, III, vol. 355, pp. 239-253, 2004.

[3] G. Higman and Q. Mushtaq, "Generators and relations for G," Arab Gulf Journal of Scientific Research, vol. 1, no. 1, pp. 159-164, 1983.

[4] O. Koruoglu, "The determination of parabolic points in modular and extended modular groups by continued fractions," Bulletin of the Malaysian Mathematical Sciences Society, vol. 2, no. 3, pp. 439-445, 2010.

[5] A. R. Seadawy, D. Lu, and C. Yue, "Travelling wave solutions of the generalized nonlinear fifth-order $\mathrm{KdV}$ water wave equations and its stability," Journal of Taibah University for Science, vol. 11, no. 4, pp. 623-633, 2017.

[6] S. N. Daoud, "Complexity of join and Corona graphs and Chebyshev polynomials," Journal of Taibah University for Science, vol. 12, no. 5, pp. 557-572, 2018.

[7] W. Burnside, Theory of Groups of Finite Order, Dover Publications, Inc., New York, NY, USA, 2nd edition, 1995.

[8] P. Cayley, "Desiderata and suggestions: no. 2. the theory of groups: graphical representation," American Journal of Mathematics, vol. 1, no. 2, pp. 174-176, 1878.

[9] H. S. M. Coxeter and W. O. J. Moser, Generators and Relations for Discrete Groups, Springer Verlag, Berlin, Heidelberg, Germany, 4th edition, 1980.

[10] W. Burnside, Theory of Groups of Finite Order, Dover Publications, Inc., New York, NY, USA, 2nd edition, 1995.

[11] W. W. Stothers, "Subgroups of the modular group," Mathematical Proceedings of the Cambridge Philosophical Society, vol. 75, no. 2, pp. 139-153, 1974.

[12] B. Everitt, "A family of conformally asymmetric Riemann surfaces," Glasgow Mathematical Journal, vol. 39, no. 2, pp. 221-225, 1997.

[13] M. D. E. Conder, "Generators for alternating and symmetric groups," Journal of the London Mathematical Society, vol. s222, no. 1, pp. 75-86, 1980.

[14] H. Whitehead, "On certain sets of elements in a free group," Proceedings of the London Mathematical Society, vol. 41, no. 2, pp. 48-56, 1936.

[15] Q. Mushtaq, Coset Diagrams for the Modular Group, D. Phil. thesis, University of Oxford, Oxford, UK, 1983.

[16] Q. Mushtaq, "Modular group acting on real quadratic fields," Bulletin of the Australian Mathematical Society, vol. 37, no. 2, pp. 303-309, 1997.

[17] P. J. Cameron, Encyclopaedia of Design Theory, pp. 1-9, 2013, Cayley Graphs and Coset Diagrams.

[18] B. Everitt, "Alternating quotients of the $(3, q, f)$ triangle groups," Communications in Algebra, vol. 6, no. 26, pp. 1817-1832, 1997.

[19] R. C. Lyndon, P. E. Schupp, R. C. Lyndon, and P. E. Schupp, Combinatorial Group Theory, Vol. 188, Springer, Berlin, Germany, 1977.

[20] G. Nebe, R. Parker, and S. Rees, "A method for building permutation representations of finitely presented groups," in Proceedings of the Finite Simple Groups: Thirty Years of the Atlas and Beyond, Princeton, NJ, USA, 2015.

[21] A. Razaq, Homomorphic Copies of Circuits in PSL(2,Z)-space, Ph.D thesis, Quaid-i-Azam University Islamabad, Pakistan, 2015.
[22] A. Torstensson, "Coset diagrams in the study of finitely presented groups with an application to quotients of the modular group," Journal of Commutative Algebra, vol. 2, no. 4, pp. 501-514, 2010.

[23] A. Razaq, Q. Mushtaq, and A. Yousaf, "The number of circuits of length 4 in PSL $(2, \mathbb{Z})$-space," Communications in Algebra, vol. 46, no. 12, pp. 5136-5145, 2018.

[24] Q. Mushtaq and A. Razaq, "Equivalent pairs of words and points of connection," The Scientific World Journal, vol. 2014, Article ID 505496, 2014. 